\title{
Iris Recognition: A Method to Increase the Robustness to Noisy Imaging Environments Through the Selection of the Higher Discriminating Features
}

\author{
Hugo Proença and Luís A. Alexandre \\ Dep. of Computer Science, IT - Networks and Multimedia Group \\ Universidade da Beira Interior, Covilhã, Portugal \\ Email: \{hugomcp,lfbaa\}@di.ubi.pt
}

\begin{abstract}
Continuous efforts have been made in searching for robust and effective iris coding methods, since Daugman's pioneering work on iris recognition was published. However, due to lack of robustness, the error rates of iris recognition systems significantly increase when images contain large portions of noise (reflections and iris obstructions), resultant from less constrained imaging conditions. Current iris encoding and matching proposals do not take into account the specific lighting conditions of the imaging environment, decreasing their adaptability to such dynamics conditions. In this paper we propose a method that, through a learning stage, takes into account the typical noisy regions propitiated by the imaging environment to select the higher discriminating features. Our experiments were performed on two well known iris image databases (CASIA and UBIRIS) and show a significant decrease of the error rates in the recognition of iris images corrupted by noise.
\end{abstract}

Keywords: Iris Recognition, Feature Selection, Biometrics.

\section{Introduction}

Iris recognition has been successfully applied in such distinct domains as airport check-in or refugee control. However, for the sake of accuracy, current systems require that subjects stand close (less than two meters) to the imaging camera and look for a period of about three seconds until the data is captured [5]. This restricts the range of domains where iris recognition can be applied, namely under natural lighting environments. In this context, the overcome of these imaging constrains has motivated the efforts of several authors and deserves growing attention by the research community. Although some of the published iris recognition approaches perform noise detection to avoid that noisy components of the biometric signatures are taken into account, we believe that highly heterogeneous lighting environments (specially under natural light) lead to the appearance of regions which, even for humans, are very difficult to classify as "noisy" or "noise-free". Figure 1 illustrates some of the noise factors that result of less constrained image capturing environments. In figure $1 \mathrm{~b}$ large iris regions obstructed by reflections (lighting and specular) can be observed, some of them very difficult to distinguish from the noise-free ones.

Moreover, traditional feature selection approaches are difficult to apply, due to the huge number of features and to their empirically proved statistical independence (Daugman [2]). Due to these facts, in this paper we propose a method that operates after the physical installation of the image 


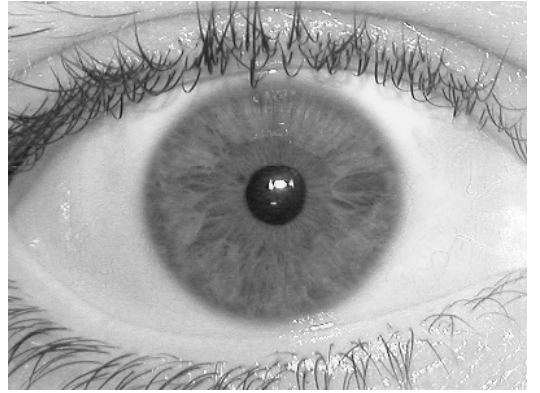

(a) Iris image with good quality.

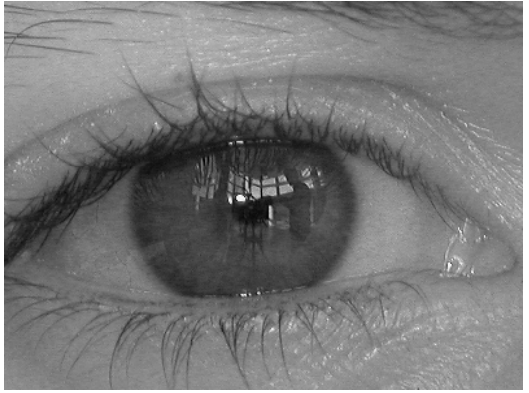

(b) Noisy iris image.

Figure 1. Comparison between a good quality image and a noise-corrupted one. Figure 1a was captured under high constrained imaging conditions and is completely noise-free. Oppositely, figure $1 \mathrm{~b}$ incorporates several types of noise that result of less constrained imaging conditions and significantly increase the challenges of the recognition task

capturing framework and selects the higher discriminating features, according to the specific imaging characteristics of the respective environment. At first, a learning set of iris images is captured, being expected that it reflects the typical characteristics (predominant noisy regions) of the images captured within the environment. Further, the candidate features are extracted from these images and used in the computation of a correspondent quality measure. This value is used as the objective function of a filter subset selection process. Our experiments showed a substantial increase in the separability between the intra- and inter-class comparisons when the $30 \%$ features with highest quality values of the learning data are selected, contributing for the increase of the recognition's robustness.

The remainder of this paper is organized as follows: section 2 briefly summarizes some of the most cited iris recognition methods. A detailed description of the proposed method is given in section 3. Section 4 reports the experiments and results and, finally, section 5 concludes this paper.

\section{Iris Recognition}

In spite of the specificities of the different proposals, typical iris recognition algorithms share the structure given in figure 2.

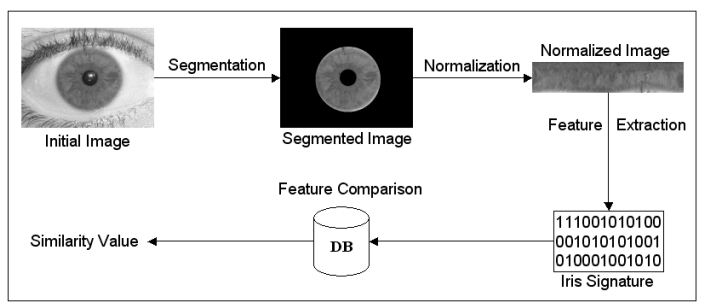

Figure 2. Typical stages of iris recognition.

The initial stage deals with iris segmentation, which is usually performed through the use of a 
rigid or deformable iris template (e.g., Daugman [2]) or through its boundary (e.g., Wildes [8]). In order to compensate the variations in pupils size and in the image capturing distances, the segmented iris region is translated into a double dimensionless polar coordinate system, through a method (Daugman Rubber Sheet) proposed by Daugman [2]. Regarding feature extraction, published approaches can be divided into three major categories: phase-based methods (e.g., [2]), zero crossing methods (e.g., [1]) and texture analysis based methods (e.g., [8]). Finally, the comparison between iris signatures is performed and produced a numeric dissimilarity value. If this value is higher than a threshold, the system outputs a non-match, meaning that each signature belongs to different irises. Otherwise, the system outputs a match, meaning that both signatures were extracted from the same iris. Here, it is usual to apply distance metrics (Hamming [2], Euclidean [3], Weighted Euclidean [6]) or methods based on signal correlation [8].

\section{Proposed Method}

The problem of feature selection is to take a set of candidate features and select a subset that best performs under some classification system, usually through the maximization of an objective function $J($.$) , either a "filter" or a "wrapper". This process reduces the cost of classification, by$ reducing the number of features that must be collected, and provides better results due to the finite sample size effects.

The rationale behind the proposed filter objective function is to valuate features that respectively maximize and minimize the dissimilarity values of the inter- and intra-class signature comparisons. Thus, the distance between feature values increases the respective quality value (1) if they were extracted from images of different irises and decreases it if the features were extracted from images of the same iris.

In the following discussion we will use $F_{i}^{p}$ to denote a feature set extracted from the $i^{\text {th }}$ iris image of the subject $p$. Also, $f_{i, j}^{p}$ denotes the $j^{\text {th }}$ feature of the $i^{t h}$ feature set extracted from the iris of subject $p, F_{i}^{p}=\left\{f_{i, 1}^{p}, \ldots, f_{i, t}^{p}\right\}$. Let $\mathbb{S}=\left\{F_{1}^{p_{1}}, \ldots, F_{n}^{p_{k}}\right\}$ be the set of learning feature sets extracted from $n$ images of $k$ subjects. The quality value $q($.$) of each candidate feature i$ is given by: $q(i):\{1, \ldots, t\} \rightarrow \mathbb{R}:$

$$
q(i)=\sum_{j=1}^{n-1} \sum_{k=j+1}^{n} \frac{\operatorname{dist}\left(f_{j, i}^{p}, f_{k, i}^{s}\right)}{\left(t_{I}-t_{E}\right) \delta_{p, s}+t_{E}}\left(1-2 \delta_{p, s}\right)
$$

where $\operatorname{dist}(.,$.$) is the function that gives the features dissimilarity (e.g., Hamming or Euclidean$ distance), $\delta_{p, s}$ is the Kronecker delta and $t_{I}$ and $t_{E}$ are the total of intra- and inter-class comparisons between elements of $\mathbb{S}$.

Let $T$ and $D$ be respectively the candidate and selected feature sets, $D \subseteq T$. Also, let |.| denote the set cardinality, such that $|T|=t$ and $|D|=d$. The feature selection filter objective function of $X=\left\{D_{1}, \ldots, D_{d}\right\}$ is represented by $J(X)$. Considering that higher values of $J($.$) indicate better$ feature sets, the problem is to find a subset $D \subseteq T$ such that $|D|=d$ and maximizes the objective function $J($.$) :$

$$
J(X)=\sum_{i=1}^{d} q\left(D_{i}\right)
$$

As above stated, it should be stressed that the proposed objective function assumes the features statistical independence, which means that $J($.$) is simply maximized by the set of the d$ features 
with highest quality values (1). In the following section we describe our experiments to find the optimal value of $d$.

\section{Experiments and Results}

In our experiments we used the recognition method described by Daugman [2], essentially due to the facts of being the basis for all the commercially deployed iris recognition systems and act as main comparison term in the iris recognition literature. We compared the error rates obtained when using the feature set with 2048 components, as originally described, and our proposal. The method starts by the iris segmentation, through an integro-differential operator that searches over the image domain for the maximum in the blurred (by a Gaussian kernel) partial derivative, regarding increasing radius of the normalized contour integral of the image along a circular arc. To compensate the varying size of the pupil and of capturing distance, we translated the images into a dimensionless polar coordinate system, according to the process known as the "Daugman Rubber Sheet" [2]. Feature extraction was accomplished through the use of bidimensional Gabor filters. The real parts of the result were truncated to zero volume (to achieve illumination invariance). For each resulting bit the sign of the real and imaginary parts from quadrature image projections were analyzed and, through quantization, assigned binary values. The feature comparison was performed through the Hamming distance.

\subsection{Data Sets}

There are presently 7 public and freely available iris image databases for biometric purposes: Chinese Academy of Sciences (CASIA) [4], Multimedia University (MMU), University of Bath (BATH), Palacký University Olomouc (UPOL), Iris Challenge Evaluation (ICE), West Virginia University (WVU) and University of Beira Interior (UBIRIS) [7].

CASIA database is by far the most widely used for iris biometric purposes. However, its images incorporate few types of noise, almost exclusively related with eyelid and eyelash obstruction, similarly to the images of the MMU and BATH databases. UPOL images were captured with an optometric framework, obtaining noise-free images with extremely similar characteristics. ICE and WVU images contain several blurred and off-angle images, which are noise factors that are out of the scope of this work. Oppositely, UBIRIS database was built with the objective of simulate noncooperative image capturing. This explains the higher heterogeneity of its images and the existence of large noisy regions (iris reflections and obstructions).

The aforementioned characteristics led us to choose UBIRIS and CASIA databases for our experiments, analyzing the recognition accuracy both in highly (UBIRIS) and less noisy (CASIA) imaging environments. We selected 400 images from each database, belonging to 40 subjects. Further, we divided each data set into two subsets. The first ones - UBIRIS $S_{t r}$ and $C A S I A_{t r}$ were used as learning data, to compute the features quality value (1) and perform feature selection. The later - UBIRIS $S_{t t}$ and $C A S I A_{t t}$ - to evaluate the recognition's accuracy. As illustrated by figures $3 \mathrm{a}$ and $3 \mathrm{~b}$, images of the UBIRIS database contain severe iris obstructions by eyelids and eyelashes in the lower and upper iris regions (regions 1) and specular and lighting reflections predominant in the left and right iris extremes (regions 2). Images of the CASIA database are less

noisy and typically contain small iris obstructions due to eyelids and eyelashes in the lower and upper iris regions (regions 1). 


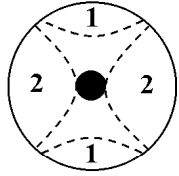

(a) Typical noisy regions in the tested iris images.

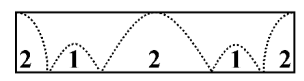

(b) Correspondent noisy regions in the segmented and normalized iris images.
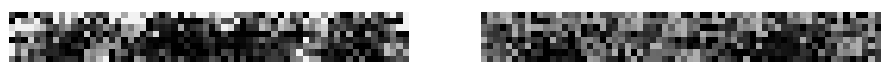

(c) Quality value of the candidate features of the $U B I R I S_{t r}$ data set. (d) Quality value of the candidate features of the $C A S I A_{t r}$ data set.

Figure 3. Correspondence between the predominant noisy regions of the data set images and the obtained features quality value (1). Brightest pixels correspond to high quality features. Note that the features quality values reflect the typical noisy regions of the correspondent data set images.

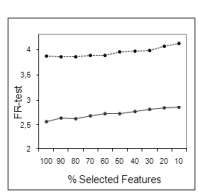

(a) FR-test values.

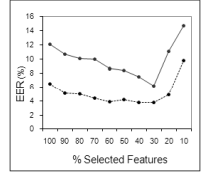

(b) Equal Error Rate (EER).

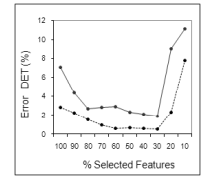

(c) Error Area Under the DET Curve.

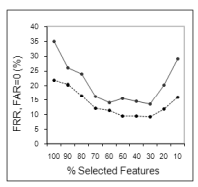

(d) FRR with FAR=0.

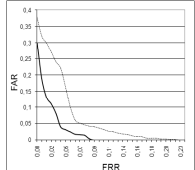

(e) DET curves obtained in the $C A S I A_{t t}$ data set

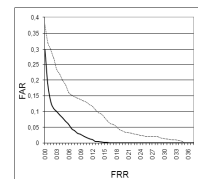

(f) DET curves obtained in the $U B I R I S_{t t}$ data set

Figure 4. Comparison between the results obtained by our proposal and the original Daugman's recognition method. The continuous and dashed lines of figures $4 a-4 d$ give the results obtained in the $U B I R I S_{t t}$ and $C A S I A_{t t}$ data sets, when varying the cardinality of the selected feature set. Figures $4 \mathrm{e}$ and $4 \mathrm{f}$ compare the DET curves obtained when using $100 \%(2048)$ and the $30 \%(614)$ features with highest quality values (1) in the biometric signatures comparison.

\subsection{Results}

According to the process described in section 3, we extracted the candidate features from all images of the learning data sets and we computed each feature's quality value (1). As the used feature extraction method (Gabor wavelets decomposition) allows the simultaneous scale-space data representation, it is possible to associate each feature with a pixel of the original data. Figures $3 \mathrm{c}$ and $3 \mathrm{~d}$ illustrate the quality of the candidate features obtained in the $U B I R I S_{t r}$ and $C A S I A_{t r}$ data sets. Here, each pixel intensity $I(p)$ is given by $\frac{q(p)-\min _{q}}{\max _{q}-\min _{q}} * 255$, where $q(p)$ is the feature quality value given by(1). For the purpose of visualization, $\min _{q}$ and $\max _{q}$ are, respectively, the minimum and maximum quality values. It can be observed that figure $3 \mathrm{~d}$ is brighter and its intensity values are more homogeneous than those of figure $3 c$ (noisiest data set). Interestingly, several dark regions can be observed, whose correspond to features that were extracted from the typical noisy regions of the learning set images and have lowest quality values. According to the proposed method, these features should not be taken into account in the comparison biometric signatures.

In order to find the optimal value of $d$, we varied the cardinality of the selected feature set between $10 \%$ and $90 \%$ of the number candidate features $(t)$. Figures $4 \mathrm{a}-4 \mathrm{~d}$ contain the results. Figure 4 a contains the values for a Fisher-ratio (FR) test given by $\tau=\left(\mu_{E}-\mu_{I}\right) / \sqrt{\frac{\sigma_{I}^{2}}{t_{I}}+\frac{\sigma_{E}{ }^{2}}{t_{E}}}$, 
where $\mu_{I}$ and $\mu_{E}$ indicate the mean of the intra- and inter-class comparisons. $\sigma_{I}$ and $\sigma_{E}$ indicate the respective standard deviation and $t_{I}$ and $t_{E}$ are the number of intra- and inter-class comparisons between iris signatures. Figures $4 \mathrm{~b}$ and $4 \mathrm{c}$ respectively contain the equal error rates (EER) and the areas of the region under the detection error tradeoff curves (DET). Finally, figure $4 \mathrm{~d}$ gives the false rejection rates when the false acceptances were minimized. In all these figures the horizontal axis give the proportion between $d$ and $t$. As the value of $d$ decreased we observed an increase in the separability between the intra- and inter- class comparisons (figure 4a). However, when $d$ is below $30 \%$ of $t$, this has no correspondence in the error rates, as the lowest error rates were obtained when $d / t=0.3$ (using 614 features). In this case we obtained an EER of about $6 \%$ in the highly noisy images of the UBIRIS data set. This must be considered an achievement, as the correspondent EER of the original method was above $12 \%$.

Finally, figures $4 \mathrm{e}$ and $4 \mathrm{f}$ compare the DET's obtained by the Daugman recognition method when using the complete feature set (dashed lines) and the 614 features (continuous lines) with highest quality features in the biometric signatures comparison. A significant decrement of the error rates can be observed, which led us to conclude that our proposal contributes for the adaptability of the recognition system to the typical image noisy regions and increases the recognition robustness to noise.

\section{Conclusions}

Under less constrained lighting environments, it is expected that the captured iris images contain several types of noise. Moreover, the predominant noisy regions are strongly determined by the environment lighting conditions. Due to the huge number of features, traditional feature selection methods are difficult to apply. According to these facts, we proposed a method that takes into account (through an images learning set) the typical characteristics of the captured images in the environment to compute a quality measure for each candidate feature.

This value was used as objective function of a feature subset selection process that determines the features that must be taken into account within the respective environment. Our experiments showed that this simple process increases the adaptability of the recognition system to the specific imaging environment. Lastly, when finding the optimal number of selected features, we obtained the lowest error rates when $30 \%$ of the highest quality features are selected (614 features). In this situation, when compared to the original recognition method, our proposal decreased the error rates over $50 \%$ in the recognition of highly noisy iris images and contributed for the recognition robustness to noise.

\section{Acknowledgements}

We acknowledge the support of the Portuguese Fundação para a Ciência e Tecnologia (FCT) and Fundo Europeu de Desenvolvimento Regional (FEDER), project PTDC/EIA/69106/2006.

\section{References}

[1] W. W. Boles and B. Boashash. A human identification technique using images of the iris and wavelet transform. IEEE Transactions on Signal Processing, vol. 46, no. 4, pages 1185-1188, April 1998. 
[2] J. G. Daugman. High confidence visual recognition of persons by a test of statistical independence. IEEE Transactions on Pattern Analysis and Machine Intelligence, Vol. 25, No. 11, pages 1148-1161, November 1993.

[3] Y. Huang, S. Luo, and E. Chen. An efficient iris recognition system. In Proceedings of the First International Conference on Machine Learning and Cybernetics, pages 450-454, China, November 2002.

[4] Institute of Automation, Chinese Academy of Sciences. CASIA iris image database, 2004.

[5] International Biometric Group. Independent test of iris recognition technology, 2005. http://Www.biometricgroup.com/reports

[6] L. Ma, Y. Wang, and T. Tan. Iris recognition using circular symmetric filters. In Proceedings of the 25th International Conference on Pattern Recognition (ICPR02), vol. 2, pages 414-417, 2002.

[7] H. Proença and L. A. Alexandre. UBIRIS: A noisy iris image database. In 13th International Conference on Image Analysis and Processing (ICIAP2005), pages 970-977, September 2005.

[8] R. P. P.Wildes. Iris recognition: an emerging biometric technology. In Proceedings of the IEEE, vol. 85, no.9, pages 1348-1363, U.S.A., September 1997. 